\title{
Entangled Titanium Fibre Balls Combined with Nano Strontium Hydroxyapatite in Repairing Bone Defects
}

\author{
Ping Liu ${ }^{a}$ d Nan Wang $^{b}$ Yongqiang Hao ${ }^{b}$ Qinghua Zhao ${ }^{c}$ Yongmin Qiao $^{a}$ \\ Hui $\mathrm{Li}^{\mathrm{a}}$ Jipeng $\mathrm{Li}^{\mathrm{C}}$ \\ ${ }^{a}$ Department of Research and Development, Shanghai Shanshan Tech. Co., Ltd., and ${ }^{b}$ Department of Orthopaedic \\ Surgery, Shanghai Ninth People's Hospital, and 'Department of Orthopaedic Surgery, Shanghai First People's \\ Hospital, School of Medicine, Shanghai Jiao Tong University, Shanghai, and d School of Chemistry, Zhejinag \\ University, Hangzhou, China
}

\section{Key Words}

Entangled titanium fibre balls · Nano strontium hydroxyapatite - Osteoconductivity - Osteoinductivity . Bone defects, repair

\begin{abstract}
Objective: To investigate the effect of entangled titanium fibre balls (ETFBs) combined with nano strontium hydroxyapatite (nano-Sr-HAP) on the repair of bone defects in vivo. Materials and Methods: Twenty-four 6-month-old, specific pathogen-free, male Sprague-Dawley rats were used. Drill defects were created in bilateral femoral condyles. ETFBs combined with nano-Sr-HAP were selected randomly from 72 samples and implanted into the femoral bone defects of left legs, which served as the experimental group, while ETFBs without nano-Sr-HAP were implanted into right legs for comparison. The bone defects on both sides were Xrayed. The anteroposterior positions and histological procedures and evaluations of each sample were performed at 1 , 2, 4 and 8 weeks post-surgery. Results: Histological results showed that the ETBs allowed new bone to grow within their structure. Additionally, an increase in new bone was seen on the nano-Sr-HAP side compared to the control side. The results of histomorphometric analysis confirmed that the new
\end{abstract}

bone formation on the left side gradually increased with time. There was a statistical increase in new bone at 2, 4 and 8 weeks, and the differences between the two sides were statistically significant at weeks 4 and 8 ( $p<0.05$ for all comparisons). Conclusion: The results showed that ETFBs possess a unique 3-dimensional interconnective porous structure and have excellent biocompatibility, cell affinity and osteoconductivity, which makes them useful as scaffold materials for repairing bone defects. On the other hand, nanoSr-HAP improved the bone defect-repairing capacity of the ETFBs, which showed osteoinductive properties.

ㄷ) 2014 S. Karger AG, Basel

\section{Introduction}

Currently, porous titanium with an adequate pore structure (i.e. a characteristic appearance similar to cancellous bone) and appropriate mechanical properties is sought as the ideal orthopaedic material for applications in hip and knee arthroplasty, bone graft substitution and spine surgery [1]. Interconnected pores are necessary for the formation of bone tissue because they allow the migration and proliferation of osteoblasts and mesenchymal cells, as well as vascularization [2]. These pores also fa-

\begin{tabular}{ll}
\hline KARGER & $\begin{array}{l}\text { () 2014 S. Karger AG, Basel } \\
1011-7571 / 14 / 0233-0264 \$ 39.50 / 0 \quad \text { Karger }\end{array}$ \\
$\begin{array}{l}\text { E-Mail karger@karger.com } \\
\text { www.karger.com/mpp }\end{array}$ & $\begin{array}{l}\text { This is an Open Access article licensed under the terms of the } \\
\text { Creative Commons Attribution-NonCommercial 3.0 Un- } \\
\text { ported license (CC BY-NC) (www.karger.com/OA-license), } \\
\text { applicable to the online version of the article only. Distribu- } \\
\text { tion permitted for non-commercial purposes only. }\end{array}$
\end{tabular}

Mr. Qinghua Zhao

Department of Orthopaedic Surgery, Shanghai First People's Hospital

School of Medicine, Shanghai Jiao Tong University

100 Haining Road, Shanghai 200080 (China)

E-Mail sawboneszhao2013@163.com 
cilitate the transport of nutrients and metabolic waste [3]. Moreover, they permit cell and tissue ingrowth, thus anchoring the prosthesis to the surrounding bone and preventing loosening of the implant.

A new type of porous material, i.e. entangled titanium fibre material or titanium fibre mesh, has excellent mechanical properties which allow it to be moulded to the required shape, and it has been proven to be bone biocompatible [4]. Used as an implant, the entangled titanium fibre material or titanium fibre mesh has osteoconductive abilities [1], but it does not reveal any osteoinductive ability. In order to improve and coordinate its mechanical, biological and chemical properties, surface modification or introduction of an osteoinductive factor is often performed [4-6]. In recent years, strontium ranelate has been found to have superior properties [716]. Strontium can enhance the precursors of osteogenic cell proliferation, directly or indirectly promote the synthesis of bone matrix and affect the microstructure and geometry of the bone through activation of the calcium-sensitive receptor of osteoblasts, representing an increase in bone strength. At the same time, it can also decrease the number of osteoclasts and reduce their activity, inhibiting bone absorption. Hence, strontium can be said to have a unique 'dual adjustment function'. The purpose of this study was to investigate the effect of entangled titanium fibre balls (ETFBs) combined with nano strontium hydroxyapatite (nano-Sr-HAP) on the repair of bone defects in femoral condyles using a rat model.

\section{Materials and Methods}

\section{Materials}

ETFBs were fabricated manually by randomly interengaging and intertwining titanium fibre (commercial purity titanium) with a diameter of $80 \mu \mathrm{m}$ (fig. 1). Its nominal chemical composition (weight \%) is: $\mathrm{Fe}=0.18, \mathrm{~N}=0.02, \mathrm{O}=0.15, \mathrm{H}=0.015$ and $\mathrm{C}=0.08$ (the balance is $\mathrm{Ti}$ ). The tensile strength of the titanium wire was $370 \mathrm{MPa}$ and the elongation was $15 \%$. The mechanical properties and behaviour of entangled titanium fibre materials have been studied and described previously [17]. The mean diameter of the ETFBs was about $2 \mathrm{~mm}$. All of the prepared ETFBs were cleaned ultrasonically for $10 \mathrm{~min}$ with deionized and distilled water and then sterilized via autoclaving for $15 \mathrm{~min}$ at $121^{\circ} \mathrm{C}$. The nano-Sr-HAP (99.99\% purity) was provided by the Instrumental Analysis Center of Shanghai Jiao Tong University, Shanghai, China. The synthesis of Sr-HAP nanostructures was carried out via the chemical precipitation method using strontium nitrate, diammonium hydrogen phosphate and ammonium hydroxide. The strontium-to-phosphorous molar ratio was similar to the hydroxyapatite stoichiometric ratio, i.e. $\mathrm{Ca} / \mathrm{P}=1.67$. nano-Sr-HAP

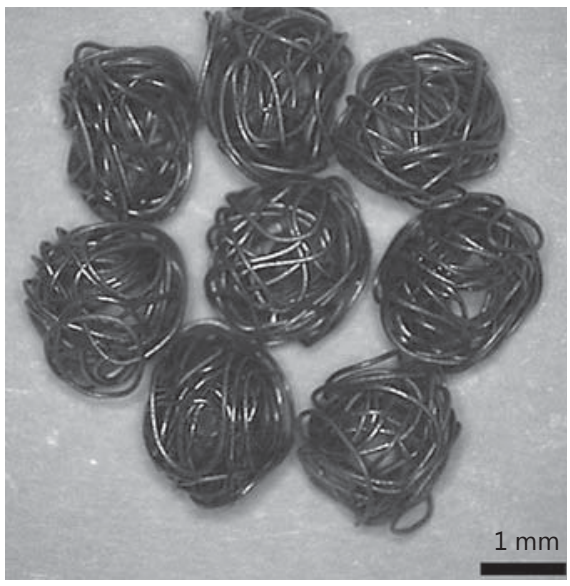

Fig. 1. Macroporous structure of the ETFBs.

exists in the crystalline phases of strontium and hydroxyapatite. The nano-Sr-HAP powder particles were spherical in shape, and their size rage was $80-100 \mathrm{~nm}$. In addition, the concentration of physiological saline used in the experiments (i.e. sodium chloride solution) was $0.9 \%$.

\section{Characteristics of the ETFBs before Implantation}

The macroporous structure of the ETFBs was observed using an XTL-500E stereomicroscope. The size of the interspaces (or voids among twisted fibres) and the size distribution of the asprepared ETFBs were determined by means of mathematical statistics, optical microscopy and image analysis as described in our previous work [18]. All samples were assessed, and 5 fields of view were chosen from the cross section of each titanium fibre ball (ETFB). In accordance with Archimedes' theory [19], liquid absorption and open porosities were determined via the hydrostatic weighing method [18]. For liquid absorption and porosity data, 95\% CI are given.

\section{Animal Model of Bone Defects and Surgery Procedure}

For implantation, twenty-four 6-month-old, specific pathogen-free, male Sprague-Dawley rats (average weight $545 \pm 22 \mathrm{~g}$ ) were used in this study following acceptance of the protocol by the relevant ethics committee. These rats were stochastically divided into 4 groups, with 6 rats in each group. They were anaesthetized by intraperitoneal injection of $20 \mathrm{~g} / \mathrm{l}$ pentobarbital sodium ( $45 \mathrm{mg} /$ $\mathrm{kg}$ ). After anaesthesia, the rats were immobilized in the lateral decubitus position. The surgical zones in the bilateral femurs of the rats were shaved, washed and disinfected with povidone iodine. Subsequently, 2 small longitudinal incisions of about $15 \mathrm{~mm}$ were made on each lateral side of the femur near the knee joint. Using blunt dissection, the femoral condyles were unveiled. The fascia was split, and drill holes $2 \mathrm{~mm}$ in diameter were made through the bilateral femoral condyles (i.e. the holes were made in both femurs, and one hole was created in each femur). All bone preparations were performed with a very gentle surgical technique using low rotational speed. Bone drill defects were created in bilateral femurs in 24 Sprague-Dawley rats. Irrigation was used to prevent thermal necrosis. 
Five grams of nano-Sr-HAP and $2.5 \mathrm{ml}$ physiological salt solution were mixed into a paste mixture so that it could evenly penetrate the interspaces of the ETFBs. Each ETFB was combined with approximately $60 \mathrm{mg}$ mixture. The implant scheme was as follows: after irrigation of the hole with saline, ETFBs combined with nanoSr-HAP were selected randomly from 72 samples and implanted into the femoral bone defects of left legs, which served as the experimental group. Also, ETFBs without nano-Sr-HAP were implanted into right legs for comparison; these served as controls. Each bone defect was filled with 3 implants. After placement of the implants, the skin was closed with staples. All rats from each group were sacrificed via an excessive dose of anaesthetic at 1, 2, 4 and 8 weeks post-surgery, respectively ( $\mathrm{n}=6$ for each implantation period). Then femurs were completely separated (a total of 48 samples were obtained), and the condition of the bone defect repairs was observed.

\section{$X$-Ray Examination}

The anteroposterior positions of each sample were observed via an X-ray (Faxitron MX20, 28KV, 14-second exposure time; USA).

\section{Histological Procedures and Evaluation}

After the X-ray, the samples were fixed in $4 \%$ paraformaldehyde, dehydrated in a series of ethanol water solutions, cleared with xylene and embedded in methyl methacrylate. At least $3 \mathrm{sec}-$ tions (initial thickness $150 \mu \mathrm{m}$ ) were prepared per sample using a hard tissue microtome (Leica SP1600; Germany) along the sagittal plane of the femur; these were then polished to a 50- $\mu$ m thickness using diamond papers and stained with picrofuchsin (Van Gieson). Finally, the sections were investigated using an optical microscope (Nikon Eclipse 80i; Japan).

Three sections from each sample, which passed through or were adjacent to the maximum cross section of ETFBs, were selected. The undecalcified sections on the sagittal plane of the femoral condyles (fig. 2) were integrally spliced using the statistical analysis system of bone histology (BioQuant OSTEO II v8.10.20; USA). New bone was selected using a unified threshold criterion $[R(255,24), G(42,8), B(67,17)]$. Then, the proportions between all of the osteogenesis volumes within the ETFBs (BV) and the total volume of ETFBs (TV) were calculated. The mean of 3 sections was taken as the trabecular bone volume (BV/TV, \%) [20].

\section{Statistical Analysis}

Statistical analysis was performed using SAS software (version 6.12). Student's t test was applied to compare the trabecular bone volume of the experimental and control groups at each time point, and $\mathrm{p}<0.05$ was considered statistically significant. $t$ values were 0.05 .

\section{Results}

\section{Characterization of ETFBs before Combination}

The macroporous structure of the ETFBs before combination is shown in figure 1 . The 3 -dimensionality and high interconnectivity of the porous structures are shown, with connective gaps both inside and on the surface of the ETFBs. The porous structures formed among the twisted

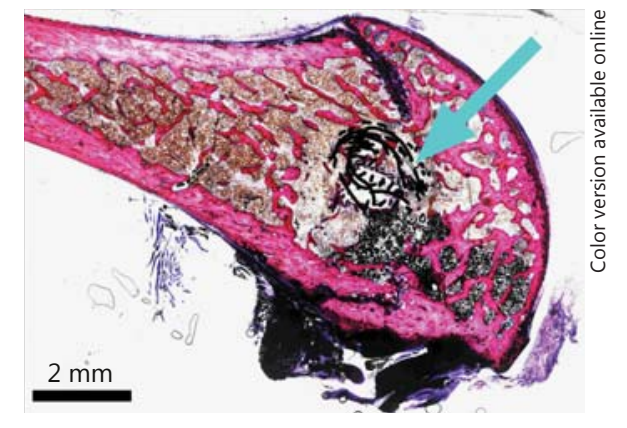

Fig. 2. Combination image of undecalcified sections on the sagittal plane of the femoral condyle. The arrow indicates the ETFBs. Picrofuchsin (Van Gieson) staining. Original magnification $\times 50$.

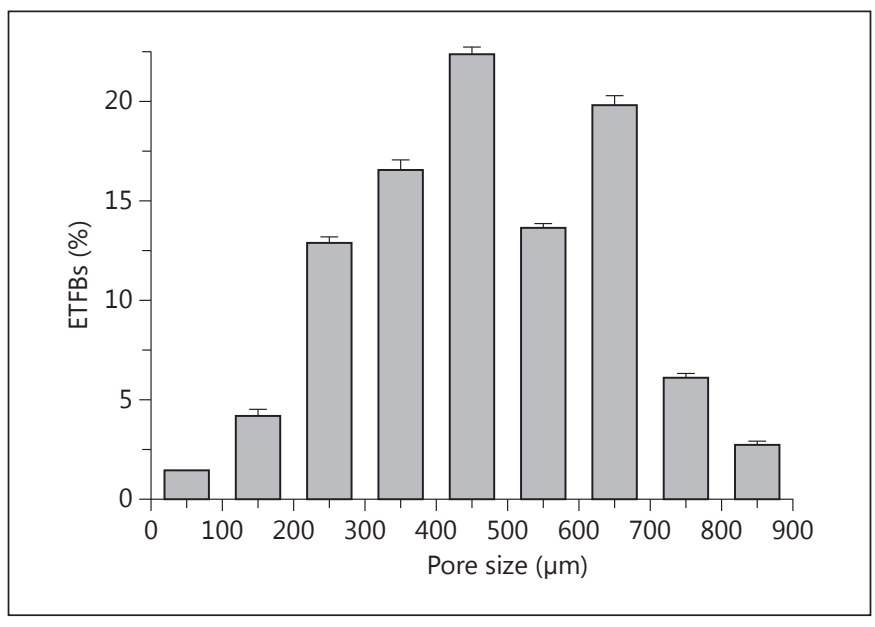

Fig. 3. Typical interspace size distribution of the ETFBs.

and crossed fibres exhibited a slot-like or irregular shape. The interspace wall was made of twisted fibres, fibre bundles and intercrossed conjunctive points. The interspace distribution was actually non-periodic. The interspace structures of the ETFBs were: open porosity and liquid absorption, $80.0 \pm 2.0 \%$ and $88.6 \pm 2.5 \%$, respectively. The typical interspace distribution in the ETFBs is shown in figure 3. Several of the sizes (95\% CI) were within the range of $100-800 \mu \mathrm{m}$, and about $1.5 \%$ of the interspaces were smaller than $100 \mu \mathrm{m}(100-500 \mu \mathrm{m}, 56.1 \%$; 500-800 $\mu \mathrm{m}, 39.6 \%$, and $800-900 \mu \mathrm{m}, 2.8 \%)$. The mean interspace size of the ETFBs was $471 \pm 27.6 \mu \mathrm{m}$.

\section{Macroscopic Clinical Findings}

All of the rats appeared to be in good health throughout the experimental period. All incisions healed well. There were no signs of hematoma, severe inflammation, 
Fig. 4. X-ray at $1,2,4$ and 8 weeks postsurgery. $\mathrm{L}=$ Experimental group (ETFBs + Sr-HAP); R = control group (ETFBs alone).

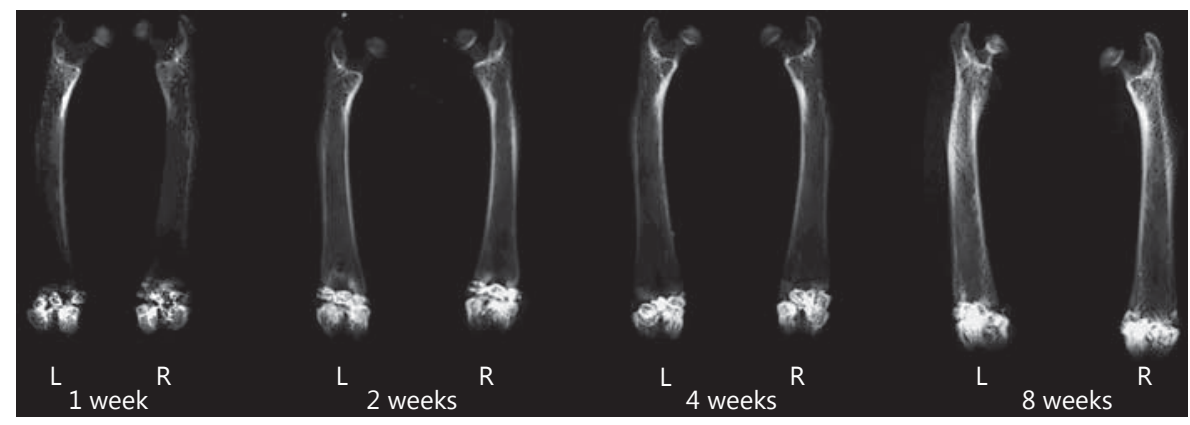

infection ulceration or other complications around the implantation sites. When all implants were explanted at the end of the various study periods, no foreign bodies were found.

\section{$X$-Ray Evaluation}

The X-ray images of the samples at 1, 2, 4 and 8 weeks post-surgery are shown in figure 4 . Bone defects implanted with ETFBs combined with nano-Sr-HAP were in left femurs, while ETFBs alone were in right ones. The defect positions in the femoral condyles of the rats had good consistency and all penetrated the bilateral cortices. Three ETFBs with high-density shadows were observed in each bone defect. On the whole, image evaluation of the repair of bone defects in the various implantation periods showed no obvious differences between the experimental and control groups. At 1 week post-surgery, the profiles of ETFBs were clear and relatively isolated, i.e. the interfaces between the ETFBs and the bones were evident. With time, the interspaces between the ETFBs, as well as the interfaces between the ETFBs and the bones gradually became blurry (fig. 4, at 2 and 4 weeks post-surgery). At 8 weeks post-surgery, the radiopaque areas in the defect zone further increased. The profiles of the ETFBs were ambiguous, and the photic zones of the interfaces between the ETFBs and the host bone disappeared.

\section{Descriptive Histological Evaluation}

At 1 week post-surgery (fig. 5a), the ETFBs in the experimental group were filled with abundant nano-SrHAP and a few were dispersed in the interstice of bone trabecula around the bone defects. Blue-stained fibrous tissues were visible around ETFBs both in the experimental group and in the control group. No obvious inflammatory cells or bone formations were observed macroscopically.

At 2 weeks post-surgery (fig. 5b), 5 of the 6 implants in the experimental group showed bone formation pene- trating through the peripheral interspaces of the ETFBs. The nano-Sr-HAP inside the ETFBs was still uniform, while the nano-Sr-HAP around the ETFBs had dissolved. Some bone formation was observed in the peripheral interspaces of ETFBs. For the control group, 3 of the $6 \mathrm{im}$ plants showed that a little bone formation penetrated through the peripheral interspaces of the ETFBs.

At 4 weeks post-surgery, newly formed bone was observed inside all of the ETFBs both in the experimental group (fig. 5c, d) and in the control group (fig. 5e, f) and it gradually grew into these ETFBs. The evolution of the newly formed bone passing through the interspaces of the ETFBs was clearly observed under a high-magnification microscope. The nano-Sr-HAP in the experimental group was degraded and absorbed to some extent. However, compared to the experiment group, less new bone formation was observed in the control group and it was distributed non-uniformly.

At 8 weeks post-surgery, abundant bone formation appeared inside the ETFBs in the experimental group (fig. 6a, b); this was statistically significantly more than in the control group (fig. 6c, d) (p value). In the experimental group, only a little nano-Sr-HAP remained. Under a high-magnification microscope, typical osteoid (or bonelike) structures were observed on the newly formed bone, i.e. osteocytes were embedded in a tissue matrix. However, some fibrous tissue existed between the new bone and the titanium fibres in the control group.

The differences in bone formation on the left side at 2 versus 4 weeks post-surgery $(t=-2.6308, p=0.0273)$ and at 4 versus 8 weeks post-surgery $(t=-2.7045, p=0.0221)$ were statistically significant. Equally, statistical evaluation revealed that the amount of bone formation inside ETFBs on the left and right sides at 4 and 8 weeks postsurgery (at 4 weeks: $\mathrm{t}=2.2417, \mathrm{p}=0.0489$; at 8 weeks: $\mathrm{t}=$ $3.5234, \mathrm{p}=0.0055$ ) was significantly larger than in the control group. 
Fig. 5. Photomicrograph of the experimental group at 1 (a), 2 (b) and 4 weeks $(\mathbf{c}, \mathbf{d})$ post-surgery. e, $\mathbf{f}$ Photomicrographs of the control group at 4 weeks post-surgery (note: these images were taken at the edge). $\mathrm{Ti}=$ Titanium fibre; $\mathrm{F}=$ nano-Sr-HAP; $\mathrm{NB}=$ new bone. Picrofuchsin (Van Gieson) staining. Original magnification $\times 50(\mathbf{a}, \mathbf{b})$, $\times 100(\mathbf{c}, \mathbf{e})$ and $\times 400(\mathbf{d}, \mathbf{f})$.

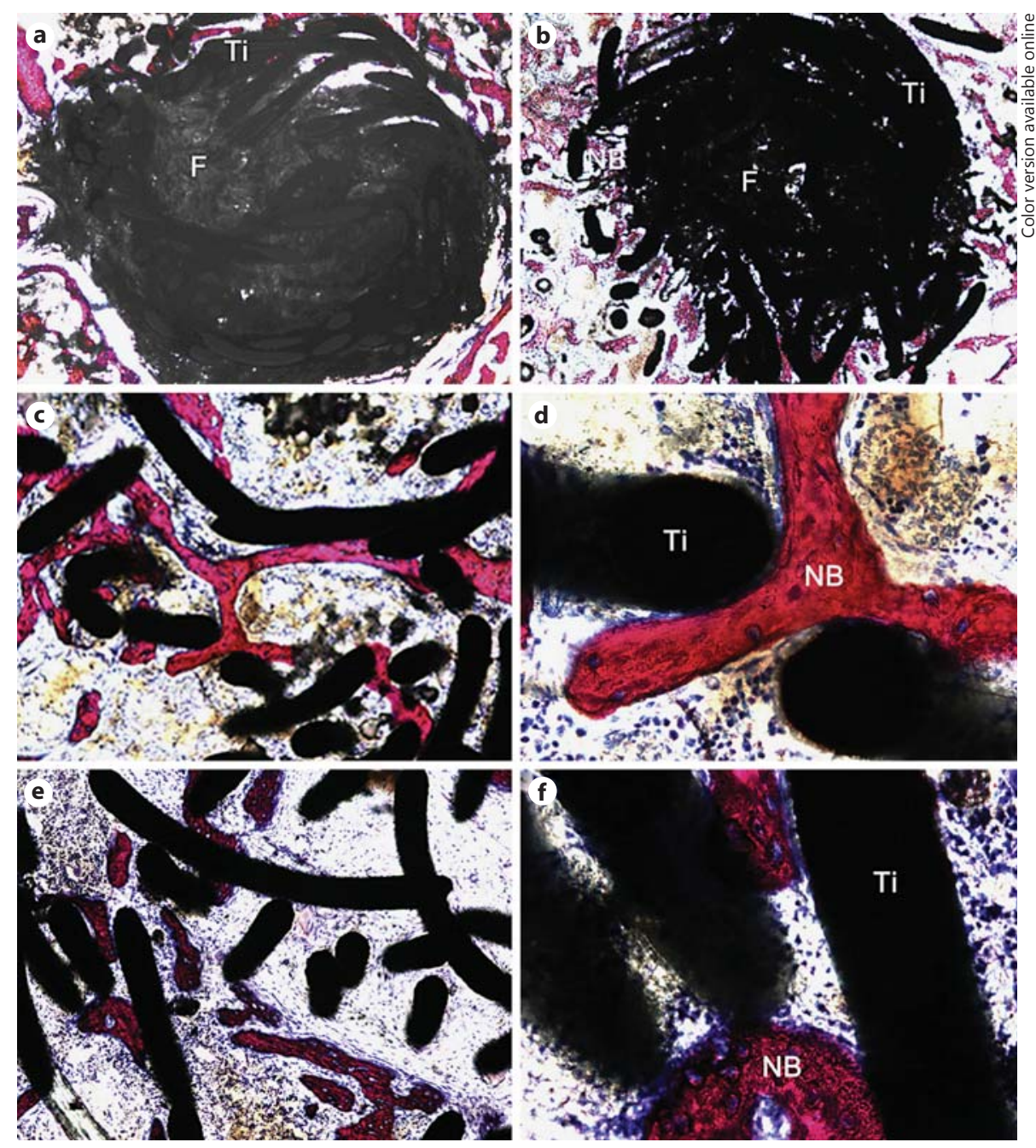

\section{Discussion}

Because the ETFBs were cleaned ultrasonically and sterilized, no signs of inflammation around the implantation sites were observed, thereby indicating that the biocompatibility of the ETFBs had not been influenced during their fabrication. Imaging showed that the interfaces between bones and ETFBs on the left and right sides gradually became blurry with time after surgery. At 8 weeks post-surgery, osteogenic bonding had occurred between the ETFBs and the host bone. Histological analysis demonstrated that the newly formed bone might have penetrated through the interspaces of the ETFBs and grown into the interior of the ETFBs (fig. 6a, b), indicating that the 100- to $800-\mu \mathrm{m}$ interspace size in ETFBs might meet the need for bone ingrowth. This finding is somewhat different from the results of the previous study [21], in which the optimal pore size for bone ingrowth ranged from 100 to $600 \mu \mathrm{m}$. This difference could be due to many factors, such as the characteristics of the sample itself, experimental conditions, the environment and methods.

Histological results demonstrated that bone formation occurred both in the experimental group and in the control group. However, the volume of bone formation in the experimental group was larger than that in the control group. The probable mechanism could be explained as follows: initially, the ingrowth of fibrous tissues around the bond defects was hindered to a certain extent because the inner interface of the ETFBs was filled with nano-SrHAP. With dissolution, a large number of strontium ions were released toward the surrounding bone tissues, which influenced the surrounding osteoblasts and osteoclasts, thereby indicating that strontium could enhance osteoblastic proliferation and differentiation and promote 
Fig. 6. Photomicrographs of the experimental (a, b) and control (c, d) groups at 8 weeks post-surgery. a Abundant bone formation was apparent inside the ETFBs. b Typical osteoid (or bone-like) structures were observed, i.e. osteocytes were embedded in a tissue matrix. c Bone formation could be observed inside the ETFBs. d Fibrous tissue existed between the new bone and the titanium fibres. Ti $=$ Titanium fibre; $\mathrm{NB}=$ new bone. Picrofuchsin (Van Gieson) staining. Original magnification $\times 100(\mathbf{a}, \mathbf{c})$ and $\times 400(\mathbf{b}, \mathbf{d})$.
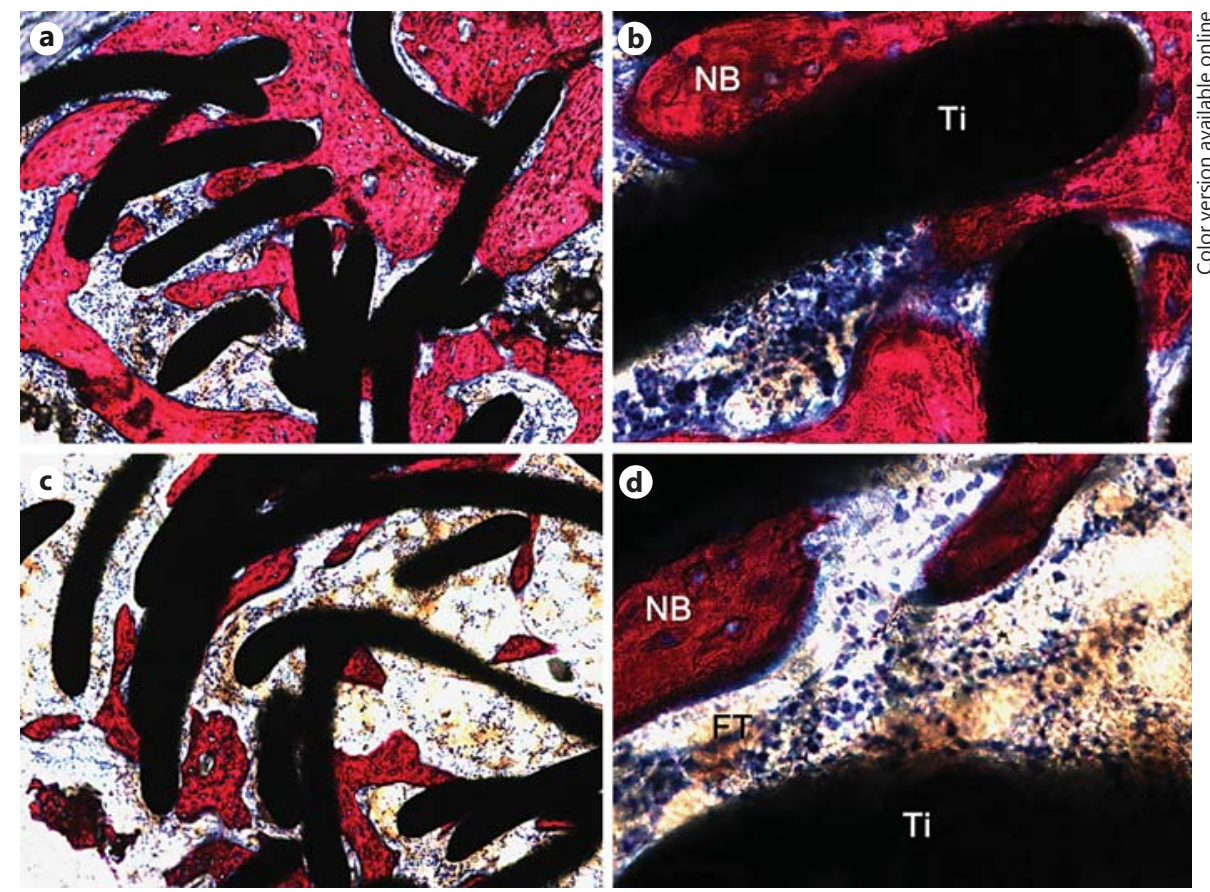

bone formation while inhibiting bone absorption. Due to a differential gradient of ion concentrations, the newly formed bone was induced to grow from the periphery to the interior of the ETFBs. At 8 weeks post-surgery, the nano-Sr-HAP had been degraded, and the whole of the ETFBs had been filled with a large number of newly formed bone islands. However, it is worth pointing out that it has been reported that, in (Ca-P)-coated mesh, bone formation starts from the centre of an interspace and proceeds in a centrifugal manner [4]. The reasons are unclear, pending further study. Subjective factors can easily interfere with the evaluation of the amount of bone formation. To avoid such possible errors, we objectively adopted a unified threshold criterion with the help of BioQuan software. Based on a statistical analysis of the volume of new bone inside each group and between the two groups at each time point, we concluded that the differences in amounts between the experimental and control groups at 4 and 8 weeks post-operatively were significant.

In this study, the implants were designed and fabricated as ETFBs with a diameter of about $2 \mathrm{~mm}$. The unique 3-dimensional interconnectivity of the porous ball structure had not only the existing predominance of titanium fibre mesh but also some other characteristics such as that: (a) the ball structure increased the surface area, which benefited the ingrowth of blood vessels and bone; (b) the ball structure also filled the variably shaped zones of bone defects, and (c) because of its smaller volume, it allowed the possibility of repairing bone defects using a minimally invasive technique. According to the China National Standard GB7314-87 method for metal compression testing, ETFBs cannot be directly tested because the size of the ETFBs is less than that of the compression test sample stipulated by the GB7314-87 method. However, we can refer to the compressive properties of entangled titanium fibre/wire materials described in our previous work [22]. The compressive properties of the entangled titanium fibre/wire materials with fibre diameters of $0.08 \mathrm{~mm}$ and a total porosity ranging from $44.2 \pm 0.1 \%$ to $46.7 \pm 0.1 \%$ (compressive plateau strength $=80.1 \pm 1.0 \mathrm{MPa}$ to 147.8 $\pm 1.8 \mathrm{MPa}$, elastic modulus $=1.4-2.3 \mathrm{GPa}$ ) were close to those of natural bone (compressive strength $=88.3-193$ $\mathrm{MPa}$, modulus of elasticity approx. 1-30 GPa).

The bioactivity of the ETFBs was improved through combination with nano-Sr-HAP, which benefited osteoblast adhesion, proliferation and differentiation and enhanced bone ingrowth and bone formation. Consequently, a firm osseointegration was established between the implant and the host bone. Two probable fixation mechanisms (mechanical interlock and chemical bonding) at the ETFB-bone interface could account for early and late fixation, thereby reducing the risk of implant loosening.

Generally, it is indicated that Sr-HAP has an X-ray radiopacity which displays a high density on the X-ray im- 
age. However, it is worth noting that Sr-HAP could not be distinguished easily in this experiment. The reason might be that Sr-HAP mostly existed at the inner interface of the ETFBs, and the amount implanted was relatively lower, so it was difficult to display owing to the restriction of the $\mathrm{X}$-ray resolution.

\section{Conclusions}

ETFBs combined with nano-Sr-HAP were used for the first time to repair drilled bone defects in this experiment. The results showed that ETFBs possess a unique 3-di- mensional interconnective porous structure and have excellent biocompatibility, cell affinity and osteoconductivity, which makes them possibly useful as scaffold materials for repairing bone defects.

\section{Acknowledgement}

This work was financially supported by the Medicine and Engineering Joint Foundation of Shanghai Jiao Tong University (contract YG2011MS28).

\section{References}

$>1$ Takemoto M, Fujibayashi S, Neo M, et al: Mechanical properties and osteoconductivity of porous bioactive titanium. Biomaterials 2005; 26:6014-6023.

-2 Kuboki Y, Takita H, Kobayashi D, et al: BMPinduced osteogenesis on the surface of hydroxyapatite with geometrically feasible and nonfeasible structures: topology of osteogenesis. J Biomed Mater Res 1998;39:190-199.

$>3$ Hutmacher DW: Scaffolds in tissue engineering bone and cartilage. Biomaterials 2000;21: 2529-2543.

4 Vehof JWM, van den Dolder J, de Ruijter JE, et al: Bone formation in Ca-P-coated and non-coated titanium fiber mesh. J Biomed Mater Res 2003;64A:417-426.

5 Fassina L, Saino E, Visai L, et al: Electromagnetic enhancement of a culture of human SAOS-2 osteoblasts seeded onto titanium fiber-mesh scaffolds. J Biomed Mater Res A 2008;87A:750-759.

6 Hayakawa T, Takahashi K, Okada H: Effect of thin carbonate-containing apatite (CA) coating of titanium fiber mesh on trabecular bone response. J Mater Sci Mater Med 2008;19: 2087-2096.

7 ParkJW, Kwon TG, Suh JY: The relative effect of surface strontium chemistry and superhydrophilicity on the early osseointegration of moderately rough titanium surface in the rabbit femur. Clin Oral Implants Res 2013; 24:706-709.
8 Park JW, Kim YJ, Jang JH, et al: Surface characteristics and primary bone marrow stromal cell response of a nanostructured strontiumcontaining oxide layer produced on a microrough titanium surface. J Biomed Mater Res A 2012;100:1477-1487.

$\checkmark 9$ Li YF, Li XD, Song GD, et al: Effects of strontium ranelate on osseointegration of titanium implant in osteoporotic rats. Clin Oral Implants Res 2011;23:1038-1044.

-10 Chung CJ, Long HY: Systematic strontium substitution in hydroxyapatite coatings on titanium via micro-arc treatment and their osteoblast/osteoclast responses. Acta Biomater 2011;7:4081-4087.

11 Han Y, Zhou JH, Zhang L, et al: A multiscaled hybrid orthopedic implant: bone ECM-shaped Sr-HA nanofibers on the microporous walls of a macroporous titanium scaffold. Nanotechnology 2011;22:1-11.

12 Vestermark MT: Strontium in the bone-implant interface. Dan Med Bull 2011;58:B4286.

13 Park JW: Increased bone apposition on a titanium oxide surface incorporating phosphate and strontium. Clin Oral Implants Res 2011; 22:230-234.

14 Maïmoun L, Brennan TC, Badoud I, et al: Strontium ranelate improves implant osseointegration. Bone 2010;46:1436-1441.

15 Park JW, Kim HK, Kim YJ, et al: Osteoblast response and osseointegration of a Ti-6Al-4V alloy implant incorporating strontium. Acta Biomater 2010;6:2843-2851.
16 Xia W, Lindahl C, Lausmaa J, et al: Biomineralized strontium-substituted apatite/titanium dioxide coating on titanium surfaces. Acta Biomater 2010;6:1591-1600.

17 He G, Liu P, Tan QB: Porous titanium materials with entangled wire structure for loadbearing biomedical applications. J Mech Behav Biomed Mater 2012;5:16-31.

18 Liu P, He G, Wu LH: Fabrication of sintered steel wire mesh and its compressive properties. Mater Sci Eng A 2008;A489:21-28.

19 Taylor RP, McClain ST, Berry JT: Uncertainty analysis of metal-casting porosity measurements using Archimedes' principle. Int J Cast Met Res 1999;11:247-257.

20 Yu XW, Xie XH, Yu ZF, et al: Augmentation of screw fixation with injectable calcium sulfate bone cement in ovariectomized rats. J Biomed Mater Res B Appl Biomater 2009;89: 36-34.

21 de Vasconcellos LMR, Leite DO, de Oliveira $\mathrm{FN}$, et al: Evaluation of bone ingrowth into porous titanium implant histomorphometric analysis in rabbits. Braz Oral Res 2010;24: 399-405.

22 Liu P, Tan QB, Wu LH, et al: Compressive and pseudo-elastic hysteresis behavior of entangled titanium wire materials. Mater Sci Eng A 2010;527:3301-3309. 